\title{
Publisher's Note: Competition between electric field and magnetic field noise in the decoherence of a single spin in diamond [Phys. Rev. B 93, 024305 (2016)]
}

P. Jamonneau, M. Lesik, J. P. Tetienne, I. Alvizu, L. Mayer, A. Dréau, S. Kosen, J.-F. Roch, S. Pezzagna, J. Meijer, T. Teraji, Y. Kubo, P. Bertet, J. R. Maze, and V. Jacques

(Received 13 June 2019; published 19 June 2019)

DOI: 10.1103/PhysRevB.99.249903

This paper was published online on 25 January 2016 with an omission of a support statement in the Acknowledgments. The Acknowledgments on page 5 should read as "We thank R. B. Liu and P. Maletinsky for fruitful discussions and careful reading of the manuscript. This research has been supported by the European Union Seventh Framework Program (FP7/2007-2013) under the project DIADEMS and by the European Research Council under the grants ERC-StG-2014, IMAGINE and ERC-CoG-2013, CIRQUSS (Grant Agreement No. 615767). J.R.M acknowledges support from Conicyt-PIA Grants No. ACT1108 and No. ACT1112, Fondecyt Grant No. 1141185, and Air Force FA9550-15-1-0113.” The Acknowledgments have been corrected as of 6 June 2019. The Acknowledgments are incorrect in the printed version of the journal. 